\title{
The Concentration of Cytosolic Free Calcium in Vertebrate Rod Outer Segments Measured with Fura-2
}

\author{
G. M. Ratto, ${ }^{1}$ R. Payne, ${ }^{1}$ W. G. Owen, ${ }^{1}$ and Roger Y. Tsien ${ }^{2}$ \\ 'Department of Biophysics and Medical Physics, and 'Department of Physiology-Anatomy, University of California, \\ Berkeley, California 94720
}

\begin{abstract}
The use of fluorescent indicators such as fura-2 (Grynkiewicz et al., 1985) to measure the cytosolic free calcium activity in retinal rods is complicated by the rods' sensitivity both to the fluorescence and to the light that excites it. By stimulating fluorescence from large numbers of rods in whole, loaded retinas and averaging repeated measurements, however, we have been able to monitor changes in free $\left[\mathrm{Ca}^{2+}\right]_{\text {, }}$ during exposure to nonsaturating lights under physiological conditions.
\end{abstract}

Retinas, isolated from the bullirog Rana catesbeiana, were loaded with fura- 2 by incubation and mounted, receptor-side up, in a perfusion chamber placed on the stage of a specially designed apparatus. A step of light delivered from above, whose wavelength alternated between 340 and $380 \mathrm{~nm}$ every $110 \mathrm{msec}$, excited fluorescence from $24 \mathrm{~mm}^{2}$ of retina and evoked a light response (the aspartate-isolated plll component of the electroretinogram-ERG). By comparing the fluorescence intensities excited by the 2 wavelengths (corrected for background and dark-noise), the free $\left[\mathrm{Ca}^{2+}\right]_{i}$ of the rod outer segment was determined.

In darkness, the $\left[\mathrm{Ca}^{2+}\right]_{\text {i }}$ of the outer segment was found to be $\sim 220 \mathrm{~nm}$. A bright light caused it to fall exponentially to $\sim 140 \mathrm{~nm}$, with a time constant of $\sim 1.6 \mathrm{sec}$. The value of $\left[\mathrm{Ca}^{2+}\right]_{i}$ at the onset of illumination was independent of stimulus intensity over a 2 log-unit range, and in all cases the fall was monotonic. After terminating the illumination, $\left[\mathrm{Ca}^{2+}\right]_{\text {, }}$ rose again to its time-zero value. These findings are consistent with the view that the $\left[\mathrm{Ca}^{2+}\right]_{1}$ is determined by the rate of influx of $\mathrm{Ca}$ through the light-sensitive channels and its rate of expulsion by the $\mathrm{Na} / \mathrm{Ca}$ exchanger.

The role of $\mathrm{Ca}^{2+}$ in the generation of the light responses of vertebrate rods is, at present, unclear. There can now be little doubt that the light-sensitive conductance is controlled by cGMP (Fesenko et al., 1985, 1986; Yau and Nakatani, 1985b), and not, as had been long supposed, by calcium ions. On the other hand, recent work has shown that both the sensitivity and kinetics of the rod response are in some way controlled by $\mathrm{Ca}^{2+}$ (Torre et al., 1986; Hodgkin et al., 1987). This has raised the possibility that $\mathrm{Ca}^{2+}$ may control the state of adaptation of the rod (Lamb, 1986; Torre et al., 1986).

\footnotetext{
Received July 8, 1987; revised Jan. 25, 1988; accepted Jan. 29, 1988.

We wish to thank T. D. Lamb, P. A. McNaughton, and A. L. Hodgkin for their many helpful comments. This work was supported by National Eye Institute Grant EY04372.

Correspondence should be addressed to Dr. W. Geoffrey Owen, Department of Biophysics, University of California, Berkeley, CA 94720.

Copyright (C) 1988 Society for Neuroscience $0270-6474 / 88 / 093240-07 \$ 02.00 / 0$
}

Two distinct pathways that facilitate the movement of $\mathrm{Ca}^{2+}$ into, and out of, the rod outer segment (ROS) have been identified. The "light-sensitive" channels are highly permeable to $\mathrm{Ca}^{2+}$ and, indeed, the inward flux of $\mathrm{Ca}^{2+}$ via this route carries $10-15 \%$ of the dark current (Yau and Nakatani, 1984; Hodgkin et al., 1985; Cervetto and McNaughton, 1986). $\mathrm{Ca}^{2+}$ is extruded from the ROS by a $\mathrm{Na} / \mathrm{Ca}$ exchanger with a stoichiometry of $3 \mathrm{Na}^{+}: 1 \mathrm{Ca}^{2+}($ Yau and Nakatani, $1985 \mathrm{a}$; McNaughton ct al., 1986; Hodgkin et al., 1987). In addition, there is evidence that indicates that $\mathrm{Ca}^{2+}$ may be sequestered from the cytosol of the ROS into endogenous stores (in particular, the disks), though exactly how much is sequestered and whether light causes any significant release into the cytosol has been disputed (Schröder and Fain, 1984; Somlyo and Walz, 1985).

An important question, which bears upon the possible modes of action of $\mathrm{Ca}^{2+}$, concerns the concentration of free, ionized calcium $\left(\left[\mathrm{Ca}^{2+}\right]_{1}\right)$ in the cytosol of the ROS. Recently, McNaughton et al. (1986) introduced the luminescent Ca photoprotein aequorin into single rods. They had been unable to measure luminescence or $\left[\mathrm{Ca}^{2+}\right]_{i}$ in normal perfusion media. However, by adding 3-isobutyl-1-methylxanthine (IBMX) to inhibit the cGMP phosphodiesterase and hence increase the light-sensitive conductance, they raised $\left[\mathrm{Ca}^{2+}\right]_{\mathrm{i}}$ sufficiently for the acquorin luminescence to be measured, and were able to demonstrate that light causes a rapid and significant reduction in $\left[\mathrm{Ca}^{2+}\right]_{\mathrm{i}}$. Furthermore, they estimated that, under normal conditions, $\left[\mathrm{Ca}^{2+}\right]_{\mathrm{i}}$ could be no greater than $\sim 0.6 \mu \mathrm{M}$. Thus, if light caused the release of $\mathrm{Ca}^{2+}$ from internal stores, as claimed by Schröder and Fain (1984), such release must not be so large as to raise $\left[\mathrm{Ca}^{2+}\right]_{\mathrm{i}}$ above that value.

In the present study, we have made use of the fluorescent calcium indicator fura-2, which can be incorporated into all the rods of a retina without mechanical disruption, and whose dissociation constant of $135 \mathrm{~nm}$ makes it better suited to the measurement of $\left[\mathrm{Ca}^{2+}\right]_{\mathrm{i}}$ in the submicromolar range. Our principal findings are that under normal conditions, $\left[\mathrm{Ca}^{2+}\right]_{\mathrm{i}}$ is $\sim 220 \mathrm{nM}$ in darkness and that moderate to bright light causes it to decline exponentially to $\sim 140 \mathrm{nM}$.

\section{Materials and Methods}

Bullfrogs were killed by decapitation and double-pithing under dim red light following overnight dark adaptation. All subsequent procedures were carried out under infrared illumination $(\lambda>850 \mathrm{~nm})$ with the aid of image converters. Eyes were dissected and retinas isolated using standard techniques. Retinas were gently washed in "calcium-free" Ringer's solution (see below) at $25^{\circ} \mathrm{C}$ before being incubated in a fresh solution of $12.5 \mu \mathrm{M}$ fura-2/AM, $0.015 \%$ Pluronic $\mathrm{F} / 27$, and $1.9 \%$ bovine serum in calcium-free Ringer's for $45 \mathrm{~min}$ at $25^{\circ} \mathrm{C}$. After a further wash in calcium-free Ringer's at $25^{\circ} \mathrm{C}$ for $15 \mathrm{~min}$, a piece of retina was mount- 
ed, receptor-side up, in a chamber that allowed both perfusion of the retina and the recording of transretinal potentials (Sickel, 1965).

Perfusion media contained $10 \mathrm{~mm} \mathrm{Na}$-aspartate to eliminate the light responses of all retinal neurons except the photoreceptors, as confirmed by recording the pIII component of the electroretinogram (ERG) (the late receptor potential). The calcium-free Ringer's solution contained $133 \mathrm{~mm} \mathrm{Na}^{+}, 2.5 \mathrm{~mm} \mathrm{~K}+1.5 \mathrm{~mm} \mathrm{Mg}^{2+}, 106.5 \mathrm{~mm} \mathrm{Cl}^{-}, 22 \mathrm{~mm}$ $\mathrm{HCO}_{3}^{-}, 10 \mathrm{~mm}$ L-aspartate, $1 \mathrm{~mm}$ pyruvate, $1 \mathrm{~mm}$ ascorbate, and $6 \mathrm{~mm}$ glucose, buffered to $\mathrm{pH} 7.6$ by bubbling with $95 \% \mathrm{O}_{2}, 5 \% \mathrm{CO}_{2}$. Normal Ringer's contained, in addition, $1.5 \mathrm{mM} \mathrm{CaCl}_{2}$. Manganese was applied by switching to normal Ringer's solution to which had been added 0.5 mM IBMX and 5 mM $\mathrm{MnCl}_{2}$.

The ultraviolet light used to excite fluorescence and to stimulate the photoreceptors was derived from a 2-beam optical stimulator with a xenon arc source, with one beam providing $340 \mathrm{~nm}$ light and the other $380 \mathrm{~nm}$ light of approximately equal radiometric intensity, and thus of equal efficiency in stimulating rods. A microprocessor-controlled shutter system was used to alternate between the 2 wavelengths every $110 \mathrm{msec}$ (see below). The collimated light passed into an otherwise light-tight Faraday cage, and was delivered to the receptor side of the retina via a 15 -mm-diameter quartz hemisphere. A circular patch, $5.5 \mathrm{~mm}$ in diameter, was thus illuminated. The irradiance was calibrated with a broadband photodiode placed in the plane of the retina (see Fig. 1)

Dye fluorescence and photoresponse were stimulated by a $5.7 \mathrm{sec}$ step of light, the wavelength of which alternated between 340 and $380 \mathrm{~nm}$ every $110 \mathrm{msec}$. Because of the finite time required for shutters to open or close, the counting of fluorescent photons was confined to a $100 \mathrm{msec}$ period beginning $8 \mathrm{msec}$ after each change of wavelength had been initiated. The counts from each of these intervals were stored on a magnetic disk. The aspartate-isolated pIII response was simultaneously recorded on frequency-modulated (FM) magnetic tape. Up to 20 such stimulations were necessary at the lowest intensities used, though fewer were required at higher intensities.

At the end of each experiment, the retina was perfused with Ringer's containing IBMX and manganese. Manganese effectively quenches fura-2 fluorescence (Grynkiewicz et al., 1985), while IBMX renders the ROS highly permeable to manganese (Capovilla et al., 1983). The retina was again stimulated and the residual (background) fluorescence measured. In several control experiments, a retina was cut in half, one-half loaded with fura- 2 , the other kept dark-adapted in oxygenated Ringer's. In each case, the residual fluorescence of the loaded half, after quenching with manganese, closely matched the fluorescence of the unloaded half, confirming the efficacy with which manganese acts.

From the several sequences of stored data, the number of fluorescent photons counted during each $100 \mathrm{msec}$ interval of stimulation was averaged. Each average included contributions from the system darknoise and retinal autofluorescence, and these were corrected for by subtracting the residual count obtained after fura- 2 fluorescence had been quenched with manganese. By comparing each corrected count with the mean of those immediately preceding and following it, the ratio $R$ $\left(=F_{340} / F_{380}\right)$ was determined as a function of time throughout the stimulation.

The internal free [Ca] was determined from the equation;

$$
\left[\mathrm{Ca}^{2+}\right]_{\mathrm{i}}=K_{\mathrm{d}} \cdot \frac{R-R_{\min }}{R_{\mathrm{atax}}-R} \cdot \frac{F_{0}}{F_{3}},
$$

where $F_{0} / F_{\mathrm{s}}$ is the ratio of fluorescent intensities evoked by $380 \mathrm{~nm}$ light from dye in solutions containing nanomolar $\mathrm{Ca}^{2+}\left([\mathrm{Ca}]_{\min }\right)$, and millimolar $\mathrm{Ca}^{2+}\left([\mathrm{Ca}]_{\max }\right) ; R_{\max }$ and $R_{\min }$ are values of $R$ at $[\mathrm{Ca}]_{\max }$ and $[\mathrm{Ca}]_{\min }$, respectively (Grynkiewicz et al., 1985). The values of these constants were determined in the usual way (see Connor, 1986, for example), using the experimental apparatus. The values were $R_{\min }=$ $0.45, R_{\max }=14.1$, and $F_{0} / F_{\mathrm{s}}=14.6$. The value of $K_{\mathrm{d}}$ was taken as 135 nм (Grynkiewicz et al., 1985).

The reliability of our values of $\left[\mathrm{Ca}^{2+}\right]_{i}$ depends on our knowing what fraction of the collected fluorescence emanates from the ROS. This, in turn, depends on a knowledge of the distribution of fura- 2 within the retina. Two separate sets of experiments were performed to determine the fraction of dye contained in the ROS. In the first, loaded, whole retinas were vigorously shaken into Ca-free Ringer's to produce a ROS suspension. Spectrophotometric comparison of the amount of rhodop$\sin$ in the suspension with that remaining in the rest of the retina indicated that, on average, $66 \pm 9 \%(\mathrm{SEM} ; n=4)$ of the outer segments

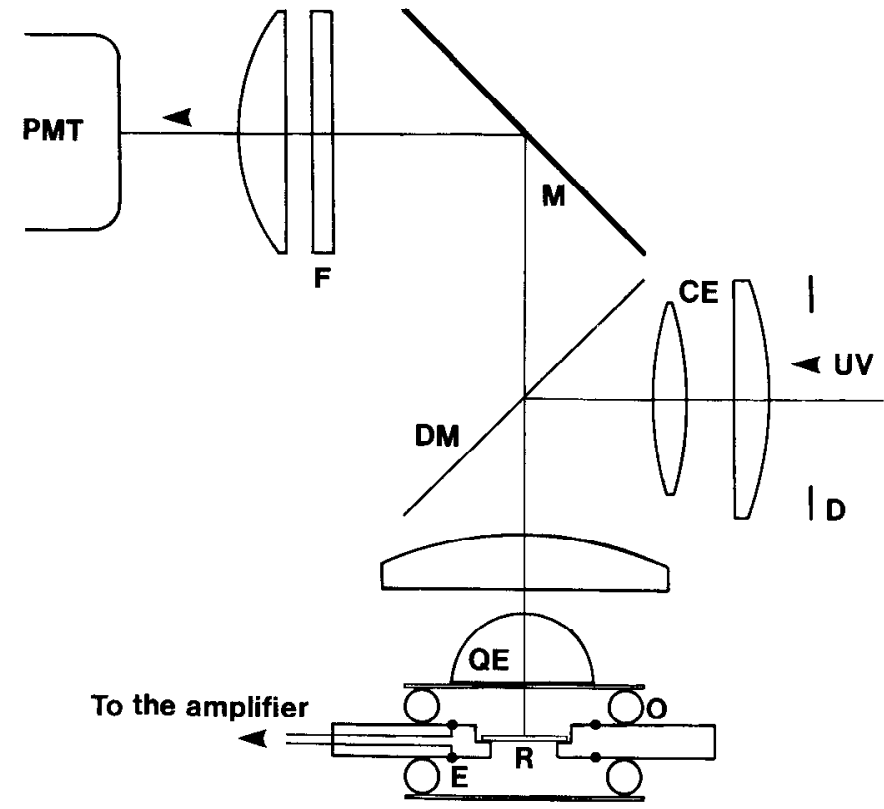

Figure 1. Schematic diagram of the apparatus. UV light entering the cage from the optical bench is first collimated, then reflected by the dichroic mirror, $D M$, onto a quartz hemisphere, $Q E, 15 \mathrm{~mm}$ in diameter. The hemisphere produces a uniform, circular patch of light, 5.5 $\mathrm{mm}$ in diameter, on the retina, $R$, which is mounted, receptor-side up, in a perfusion cell equipped with $\mathrm{Ag} / \mathrm{AgCl}$ electrodes, $E$, for the recording of transretinal potentials. The $500 \mathrm{~nm}$ fluorescence excited by the UV light is collected and collimated by the quartz hemisphere and passes through the dichroic mirror to the cathode of a cooled photomultiplier, $P M T$. The numerical aperture of the system is 1.1. Other components: $D$, diaphragm; $C E$, collimating lenses; $M$, mirror; $F$, weak lens system; $O$, neoprene o-rings.

had been shaken off. Cuvette spectrofluorimetry showed that $45 \pm 7 \%$ of the fura-2 was in the suspension. On the assumption that all dye in the suspension originated from the outer segments, which would require that inner segments reseal quickly after their outer segments become detached and do not themselves become detached from the retina during shaking, $68 \%$ of the total dye in the retina must be located in the outer segment. This should be regarded as the upper limiting value.

To ascertain how the dye was partitioned between disks and cytosol in the outer segments, the suspension was subjected to a series of treatments designed to disrupt the plasma membranes but leave the disks intact (Szuts and Cone, 1977). Rod suspensions were triturated through an 18-gauge syringe needle, pelleted by centrifugation $(13,000 \times g ; 2$ min), resuspended in fresh "Ca-free" Ringer's with further trituration, and centrifuged again. The pellet was finally resuspended in a hypotonic solution of Ca-free Ringer's and again centrifuged (13,000 $\times g ; 4 \mathrm{~min})$. The 3 resulting supernatants contained, on average, $87 \%$ of the total fura- 2 in the suspension ( $80 \%$ in the isotonic Kinger's, $7 \%$ in the hypotonic Ringer's). The remaining $13 \%$ was recovered only after digesting the pellet with Triton X-100. We conclude from these measurements that up to $\sim 68 \%$ of the fura- 2 in the loaded retina is in the ROS and that, of this, $87 \%$ is in the cytosol and $13 \%$ in, or bound to, the disks. The remaining $32 \%$ of the fura- 2 is located elsewhere in the retina.

It is worth noting that the ratio of the cytosolic volume to intradiskal volume of the frog ROS is $\sim 7.5$ (Fein and Szuts, 1982). The estimated distribution of dye between these 2 compartments suggests that it may be equally concentrated throughout the ROS. If the dye were equally concentrated in the inner segments as well, the free volume of the rod inner segment is such that virlually all of the remaining dye would be accounted for, i.e., all of the dye would be located in the rods. When one recognizes that the total surface area of the ROS is more than 25 times the planar surface area of the retina, this does not seem unreasonable.

An independent estimate of the fraction of dye contained in the ROS was obtained in a second experiment. A loaded retina was sandwiched between annular pieces of nonfluorescent Millipore filter and mounted, 
receptor-side up, in the chamber. Fluorescence was stimulated and measured in the usual way. The chamber was next inverted so that the retina was receptor-side down, and the measurement repeated. The retina was then perfused with IBMX and $\mathrm{Mn}^{2+}$ to quench the fura-2 fluorescence, and a measurement of the residual fluorescence was made. Finally, the chamber was inverted once more so that the retina was again receptorside up, and the residual fluorescence was measured. After subtracting the residual fluorescence from the total fluorescence in each configuration, we found that the measured fura-2 fluorescence was 2.1 times greater with the receptor-side down than with the receptor-side up. Taking into account the screening of both stimulating light and fluorescence by the rhodopsin in the ROS, this result indicates that about $64 \%$ of the total dye was located in the outer segments, a value close to that obtained in the fractionation experiments described above.

The concentration of dye loaded in the outer segments was estimated by 2 different techniques. An upper limit of $77 \mu \mathrm{M} \pm 17$ (SEM; $n=4)$ was obtained by assuming that all the dye shaken from the retinas (see above) was derived from the ROS. Estimates of the rhodopsin content of the rod suspension were used to calculate the ROS volume shaken from the retina on the basis of dimensions provided by Fein and Szuts (1982). A lower limit of $37 \mu \mathrm{M} \perp 12(\mathrm{SEM} ; n=4)$ was obtained by layering ROS suspensions onto a discontinuous Percoll gradient and collecting intact ROS according to the method of Hamm and Bownds (1986). ROS suspensions were layered onto a gradient of $0.5 \mathrm{ml}$ of $68 \%$ Percoll in Ca-free saline, $1 \mathrm{ml}$ of $42 \%$ Percoll in Ca-free saline, and 0.25 $\mathrm{ml}$ of $6 \%$ Percoll in Ca-free saline. The gradient was centrifuged for 5 min at $2900 \mathrm{rpm}$ on a swinging bucket rotor in a desktop centrifuge (model CL; International Clinical Centrifuge). Intact ROS (as judged by didansyl cysteine staining), very few of which had inner segments attached, collected at the boundary between Ca-free Ringer's containing $42 \%$ and $68 \%$ Percoll. Removal of this layer and assay of its fura- 2 and rhodopsin contents yielded the above lower limit to the fura- 2 concentration in the ROS, on the assumption that rods had not leaked fura-2 during resealing and/or centrifugation. Since we did not assay the total amount of dye loaded in these 2 sets of experiments, we cannot say whether the difference in these 2 values reflects different degrees of dyeloading or different amounts of dye leakage in the 2 protocols. They confirm, however, that, under normal loading conditions, the concentration of fura- 2 in the ROS is likely to be in the range 37-77 $\mu \mathrm{M}$.

Bearing in mind that, at these concentrations, the optical density of fura- 2 at $340 / 380 \mathrm{~nm}$ is $<4 \%$ that of the rhodopsin in the outer segment, and that both the stimulating light and the fluorescence are strongly absorbed by rhodopsin, it is clear that dye molecules in the ROS are more likely to contribute to the collected fluorescence than are molecules in the deeper layers of the retina. Indeed, if, as our measurements indicate, the ROS contain two-thirds of the loaded dye, we calculate that about $90 \%$ of the detected fluorescence is emitted by the dye in the outer segments. (It is worth noting that if the outer-segment dye fraction were only one-half of that amount, it would still contribute $67 \%$ of the measured fluorescence.) Essentially, rhodopsin serves as a screening pigment, helping to restrict the signal to that from the outermost portion of the retina, the outer segments, just as phenol red can be used in squid axons to confine aequorin measurements to the periphery of the axon (Mullins and Requena, 1979).

In calculating $\left[\mathrm{Ca}^{2+}\right]_{\mathrm{i}}$ from our data, we ignore the origin of the fluorescence we collect, treating all photons as though they emanate from the ROS. To do otherwise would require precise knowledge both of the distribution of dye and of the calcium levels in other dye-containing compartments, which we do not have. This must inevitably limit the reliability of our calculated values. If we assume that $\left[\mathrm{Ca}^{2+}\right]_{i}$ in the other compartments is of the same order as that in the ROS, however, then ignoring the source of the collected fluorescence would reduce our calculated values below the truc ones by about $2.5 \%$.

We considered the possibility that radiationless fluorescence energy transfer (see Lakowicz, 1983, for review) might occur between dye molecules and nearby rhodopsin molecules, since this would diminish the fraction of outer-segment dye contributing to the collected fluorescence. The efficiency of this type of energy transfer is given by

$$
E=\frac{R_{0}{ }^{6}}{R_{0}{ }^{6}+r^{6}}
$$

where $r$ is the actual distance between donor and acceptor sites on the 2 molecules, and $R_{0}$ (the Förster distance) is the characteristic distance over which energy transfer is $50 \%$ efficient. From the emission spectra of the dye and the absorbance spectrum of rhodopsin, and assuming random orientation of the dye molecules but preferential excitation of those with transition moments perpendicular to the long axis of the rod, one can calculate values for $R_{0}$ of $4.5 \mathrm{~nm}$ and $4.8 \mathrm{~nm}$, respectively, for Ca-free and Ca-bound fura-2 to rhodopsin. Steinemann and Stryer (1973) showed that when rhodopsin in intact disks was covalently labeled on its cytoplasmic face with impermeant energy donors of similar $R_{\mathrm{o}}(4.5-5.1 \mathrm{~nm})$, the energy transfer efficiency, $E$, to thodopsin was $<5 \%$, suggesting that the chromophore was buried far from the cytoplasmic face. Since probes bound to the outside faces of disks show so little energy transfer to rhodopsin, it is unlikely that fura- 2 dissolved in the cytosol would transfer significant energy either. On the other hand, the remoteness of the chromophore from the cytoplasm may indicate its proximity to the disk lumen, which is only about $2 \mathrm{~nm}$ across. Quenching of intradiskal dye by the chromophore of rhodopsin may thus be quite efficient, which would further discriminate in favor of cytosolic, as opposed to intradiskal, dye.

\section{Results}

\section{Measurement of free $\left[\mathrm{Ca}^{2+}\right]_{i}$ in the presence of $\mathrm{IBMX}$}

IBMX is a powerful inhibitor of the rod phosphodiesterase (Beavo et al., 1970; Capovilla et al., 1982, 1983). When applied in submillimolar quantities, it causes a rise in intracellular cGMP, which, in turn, leads to an increase in the light-sensitive conductance. Since the light-sensitive conductance is highly permeable to $\mathrm{Ca}^{2+}$ (Yau and Nakatani, 1984; Hodgkin et al., 1985), the free $\left[\mathrm{Ca}^{2+}\right]_{\mathrm{i}}$ rises. This effect of IBMX was used by McNaughton et al. (1986) to raise $\left[\mathrm{Ca}^{2+}\right]_{i}$ to a level at which it promoted measurable luminescence in single, aequorin-loaded rod outer segments.

In the present study, the rise of free $\left[\mathrm{Ca}^{2+}\right]_{\mathrm{i}}$ during application of IBMX was monitored directly, using fura-2, as illustrated in Figure 2. The triangles in Figure $2 A$ plot the photon-counting rate during $340 \mathrm{~nm}$ illumination, averaged over each complete stimulus train, while the circles plot the average rate during 380 $\mathrm{nm}$ illumination. These are raw values, uncorrected for background.

Initially, the count rates evoked by the 2 wavelengths were roughly equal. Following application of $0.5 \mathrm{mM}$ IBMX, however, a marked increase in the $340 \mathrm{~nm}$ count rate and a corresponding drop in the $380 \mathrm{~nm}$ rate were seen, indicating a shift in the excitation spectrum of the indicator towards shorter wavelengths and, therefore, a rise in $\left[\mathrm{Ca}^{2+}\right]_{i}$. Subsequent addition of $5 \mathrm{mM} \mathrm{Mn}^{2+}$ to the bathing medium quenched the fura-2, yielding a measure of the background fluorescence. After subtracting the background counts, the ratio $F_{340} / F_{380}$ was used to calculate the average $\left[\mathrm{Ca}^{2+}\right]_{\mathrm{i}}$ during each stimulation (Fig. $2 B$ ). It is clear that following application of the IBMX, the fura- 2 was rapidly saturated, indicating that free $\left[\mathrm{Ca}^{2+}\right]_{\mathrm{i}}$ had risen to greater than $\sim 10$ $\mu \mathrm{M}$.

Figure $2 C$ plots the change in free $\left[\mathrm{Ca}^{2+}\right]_{\mathrm{i}}$ that occurred in the course of the light response during exposure to IBMX. These data are averaged from the 7 stimulations indicated by the bar in Figure $2 A$. From an initial high value, $\left[\mathrm{Ca}^{2+}\right]_{\mathrm{i}}$ fell steadily to about $2.5 \mathrm{~mm}$, and was still falling when the stimulus was extinguished. These results are in qualitative agreement with those reported carlicr by McNaughton et al. (1986) on the basis of aequorin measurements in rods of the tiger salamander.

\section{Measurements made during perfusion with normal Ringer's solution}

When the retina was bathed in normal Ringer's solution, the free $\left[\mathrm{Ca}^{2+}\right]_{\mathrm{i}}$ of the ROS was found to fall with an approximately exponential time course during illumination. This is clearly illustrated in Figure 3. The raw counts obtained during 340 and 

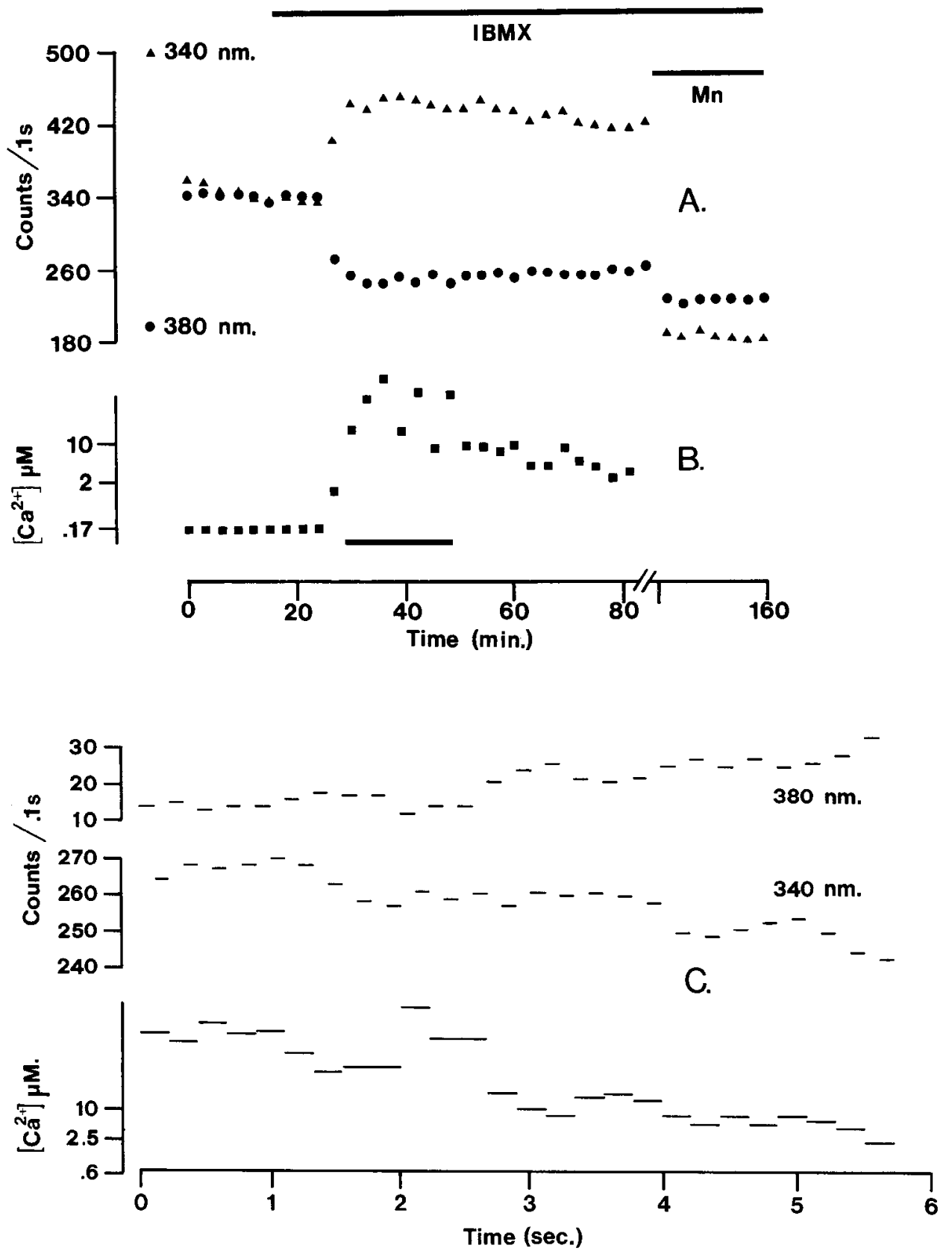

Figure 2. Effect of IBMX upon free $\left[\mathrm{Ca}^{2+}\right]_{\mathrm{i}} \cdot A$, Raw counts evoked by trains of alternating 340 and $380 \mathrm{~nm}$ light during perfusion with $0.5 \mathrm{~mm}$ IBMX. Stimuli were sufficiently intense to bleach 890 rhodopsin molecules ${ }^{-1}$ rod $^{-1}$ per $\sec ^{-1}$. Each point is the average number of fluorescent photons counted/100 msec counting interval during the $5.7 \mathrm{sec}$ of stimulation. The background count was determined by quenching the fura-2 with $5 \mathrm{~mm}$ manganese $(M n)$. $B$, Average free $\left[\mathrm{Ca}^{2+}\right]_{\mathrm{i}}$ during each stimulation, calculated from the data in $A$. Above $\sim 10 \mu \mathrm{M}$, the fura-2 was fully saturated. Because the 380 $\mathrm{nm}$ signal is so small when the dye is saturated, any variability in either background or signal causes a wide scatter in the calculated values of free $\left[\mathrm{Ca}^{2+}\right]_{i} . C$, Effect of light on free $\left[\mathrm{Ca}^{2+}\right]_{i}$ during exposure to IBMX. These data were averaged from the 7 stimulations above the bar in $B$. The counts have been corrected for background. The $\left[\mathrm{Ca}^{2+}\right]_{\mathrm{i}}$ scale is not meaningful above about $10 \mu \mathrm{M}$.
$380 \mathrm{~nm}$ illumination are shown in Figure $3 A$. The rise in the $380 \mathrm{~nm}$ counts and corresponding fall in $340 \mathrm{~nm}$ counts signify a shift in the excitation spectrum of fura- 2 towards longer wavelengths and, hence, a fall in $\left[\mathrm{Ca}^{2+}\right]_{\mathrm{j}}$.

The $\left[\mathrm{Ca}^{2+}\right]_{i}$, calculated from these data after correction for background, is plotted as a function of time in Figure $3 \mathrm{~B}$. At the onset of illumination, the $\left[\mathrm{Ca}^{2+}\right]_{i}$ was about $250 \mathrm{~nm}$ in this case, and declined exponentially during stimulation to about $165 \mathrm{~nm}$, with a time constant of $1.6 \mathrm{sec}$.

The intensity of the stimulus used to obtain these data was sufficient to saturate the rod's response, bleaching about 3400 rhodopsin molecules/sec in each rod. We carried out similar measurements using lower stimulus intensities, and the results of one such experiment on a different retina are plotted in Figure 4. The lowest intensity in this case bleached about 70 rhodopsin molecules/sec in each rod, enough to produce $70 \%$ saturation of the pIII component of the ERG. (When lower intensities than this were used, thermal and photon noise became dominant, and excessive averaging was required to produce meaningful data.) All 3 intensities caused the free $\left[\mathrm{Ca}^{2+}\right]_{\mathrm{i}}$ to decline by about the same amount, from $\sim 240$ to $-165 \mathrm{~nm}$. While the rate of decline was somewhat faster at the higher stimulus intensities, the magnitude of the decline was about the same, given the scatter in the data.

Because it is about 25 times more likely that a photon from our stimulus beam will bleach a rhodopsin molecule than excite a dye molecule, any attempt to measure the resting (dark) level of free $\left[\mathrm{Ca}^{2+}\right]_{i}$ will inevitably perturb it. A value can only be obtained by extrapolating back to the moment of stimulus onset (time zero), provided that, in addition to the slow, light-induced changes we measure, light does not also cause a significant, rapid release (or uptake) of $\mathrm{Ca}^{2+}$ from an endogenous store. One reason 
Figure 3. Effect of light on free $\left[\mathrm{Ca}^{2+}\right]_{\mathrm{i}}$ under normal conditions. The stimulus bleached $\sim 3400$ rhodopsin molecules $\operatorname{rod}^{-1} \sec ^{-1} . A$, Raw fluorescence count evoked by 340 and $380 \mathrm{~nm}$ light as a function of time during the light response. $B$, Light-induced change in free $\left[\mathrm{Ca}^{2+}\right]_{i}$, calculated from the above data after correction for background.

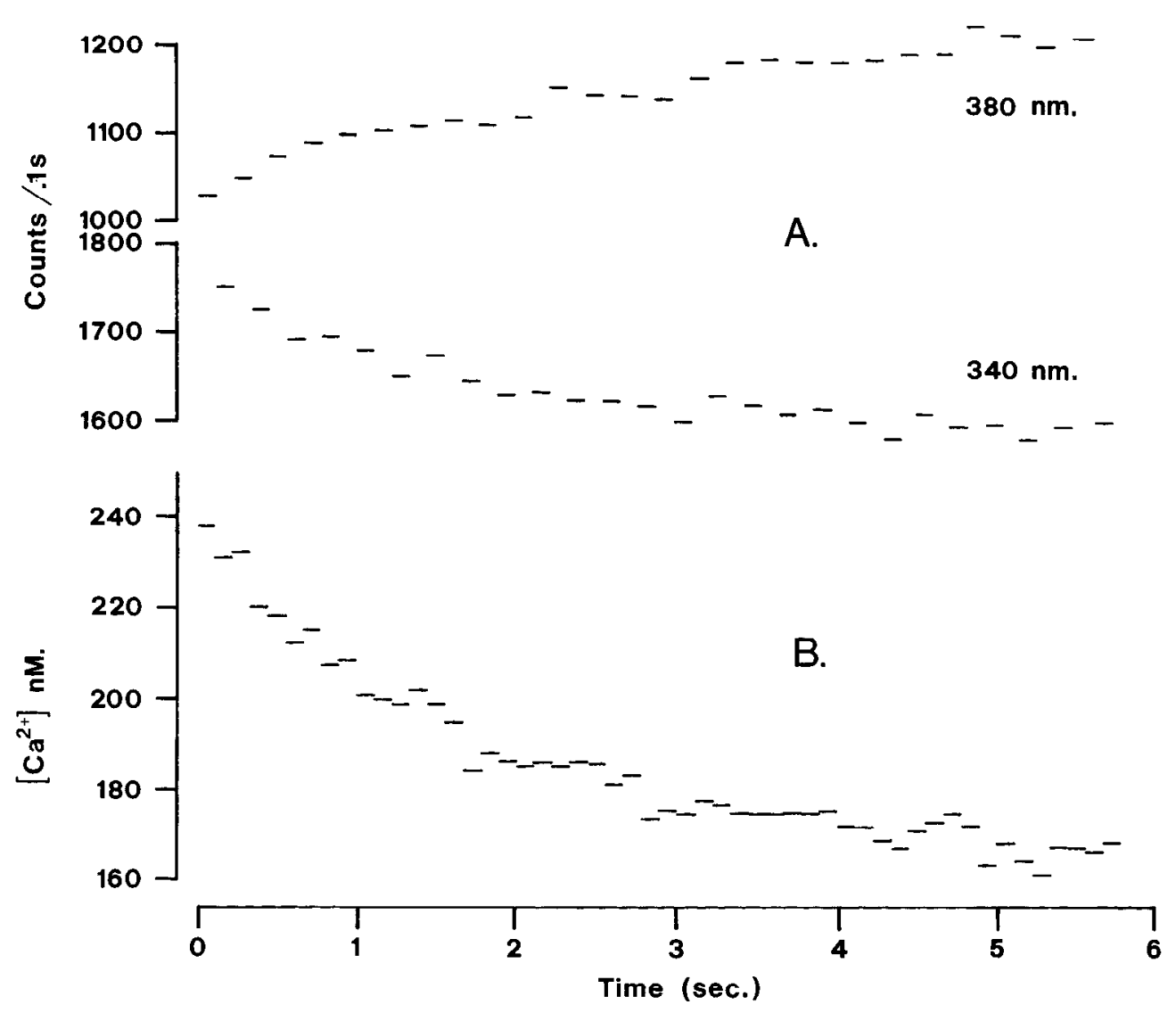

for believing that any such release is insignificant is that the resting value of free $\left[\mathrm{Ca}^{2+}\right]_{i}$ obtained by extrapolation to time zero is not intensity-dependent. This is evident both in Figure 4 and in the small variability in the mean resting value computed from all our data (see below), despite the use of a 2 log-unit range of stimulus intensities. A second reason is that, upon extinguishing the stimulus, the free $\left[\mathrm{Ca}^{2+}\right]_{\mathrm{i}}$ was found to rise once more to its initial resting value. This is shown in Figure 5.

In this experiment, the usual $5.7 \mathrm{sec}$ flash train was followed at intervals of $30 \mathrm{sec}$ by pairs of $340 / 380 \mathrm{~nm}$ flashes, which allowed us to monitor the recovery of free $\left[\mathrm{Ca}^{2+}\right]_{\mathrm{i}}$ in "darkness." Ten such sequences were presented and the values averaged. Each of the flash pairs reexcited the rods, of course, and slowed the recovery of free $\left[\mathrm{Ca}^{2+}\right]_{i}$. The apparent rate of recovery thus measured, therefore, is not meaningful. The direction of recovery is clearly revealed, however, and it can be seen that, after about $2 \mathrm{~min}$, free $\left[\mathrm{Ca}^{2+}\right]_{i}$ had returned to its resting (time zero) level. This result would require that any significant light-induced release of $\mathrm{Ca}^{2+}$ into the cytosol from endogenous stores should occur instantaneously at stimulus onset and be independent of the stimulus duration.

Taken together, these 2 findings argue against any significant light-induced release of $\mathrm{Ca}^{2+}$ from endogenous storcs. The most likely explanation of the observed fall in $\left[\mathrm{Ca}^{2+}\right]_{\mathrm{i}}$ during illumination is that it reflects the reduction in $\mathrm{Ca}^{2+}$ influx through the light-dependent channels.

The average value of free $\left[\mathrm{Ca}^{2+}\right]_{i}$ at the onset of illumination (resting level) was $221 \pm 9.5 \mathrm{~nm}$ (SEM). This average was calculated from data collected in 10 different experiments using stimulus intensities from $70 \mathrm{Rh}^{*}$ sec ${ }^{1} \operatorname{rod}^{-1}$ to $7500 \mathrm{Rh}^{*} \mathrm{sec}^{-1}$ $\operatorname{rod}^{-1}(\mathrm{Rh}=$ rhodopsin $)$. The small variability of the value thus determined is consistent with our finding that the value is not dependent on the intensity of the stimulus used to make the measurement. The average value of free $\left[\mathrm{Ca}^{2+}\right]_{i}$ reached by the end of stimulation was $140 \pm 14.5 \mathrm{~mm}$ (SEM).

\section{Discussion}

Until now, essentially all of the many applications of the fluorcscent $\mathrm{Ca}^{\text {?+ }}$ indicators quin- 2 and fura- 2 have becn in ncarly colorless tissues. At first glance, these dyes would seem to be unusable in rods, whose photosensitivity is maximal around the same wavelengths as the dyes' emission spectra. Indicators working at deep red or infrared wavelengths would be better suited, since they offer complete independence of actinic and measuring illumination. Nevertheless, the strong absorbance of rhodopsin is turned to advantage in our experiments, since it prevents most of the exciting UV or emitted green light from reaching or leaving the inner segments and deeper layers of the retina. Rhodopsin may also quench the small amounts of intradiskal dye by radiationless energy transfer. For these reasons, most of the measured dye fluorescence comes from the outer segments, even though the entire retina is exposed to the permeant ester of fura- 2 . Because a single photomultiplier collects light from all of the $\sim 4 \times 10^{5}$ photoreceptors within the illuminated patch of retina, we were able to lower illumination intensities to within the physiological working range of the rods. The absorbance of the fura- 2 is $<4 \%$ that of rhodopsin, even in the UV, and since fura- 2 has a maximal quantum efficiency of about 0.5 , reemitted light from fura- 2 adds $<2 \%$ to the excitation of rhodopsin. Optical feedback from the indicator to the rods is thus negligible. The exclusion of fluorescence from unwanted compartments cannot be perfect, however, so the $\left[\mathrm{Ca}^{2+}\right]_{i}$ changes in the outer-segment cytosol may actually be 


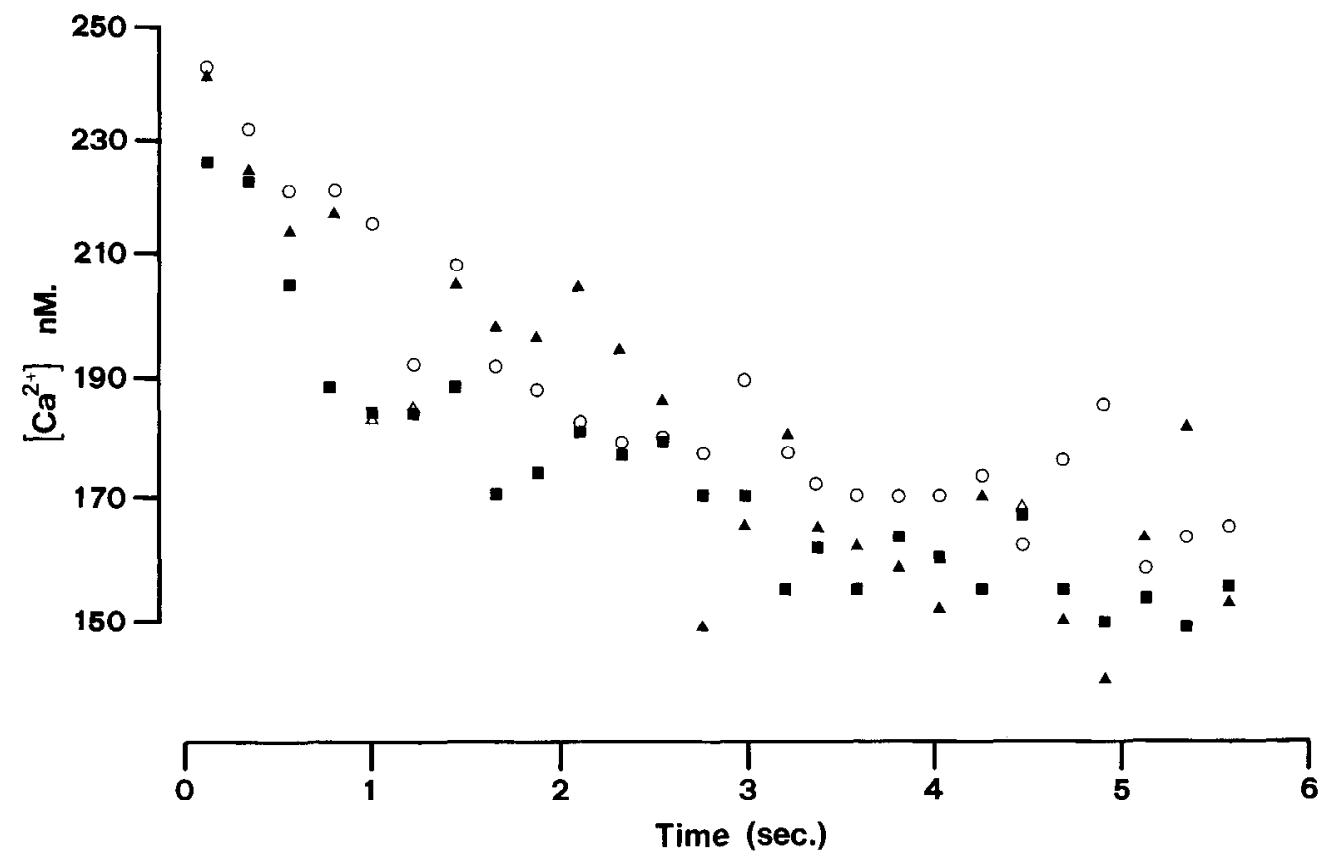

Figure 4. Light-induced change in free $\left[\mathrm{Ca}^{2+}\right]_{i}$ measured with UV light of 3 different intensities. $\mathbf{A}$, Twenty photons ${ }^{-1}$ $\sec ^{-1} \mu \mathrm{m}^{-2}$. O, Two hundred photons ${ }^{-1}$ $\sec ^{-1} \mu \mathrm{m}^{-2}$. $\sec ^{-1} \mu \mathrm{m}^{-2}$. The lowest intensity bleached $\sim 70$ rhodopsin molecules $^{-1}$ $\operatorname{rod}^{-1} \sec ^{-1}$ somewhat larger than reported here (see Materials and Methods). Our results, which constitute the first measurements of normal, resting cytosolic-free $\left[\mathrm{Ca}^{2+}\right]$ in rods, may be summarized as follows: In darkness, we found the $\left[\mathrm{Ca}^{2+}\right]_{\mathbf{i}}$ of the frog rod's outer segment to be $\sim 220 \mathrm{nM}$. A bright light caused it to fall exponentially to about $140 \mathrm{nM}$, with a time constant of $\sim 1.6$ sec. The value of $\left[\mathrm{Ca}^{2+}\right]_{i}$ at the onset of illumination was independent of stimulus intensity over a 2 log-unit range, and in all cases the fall in $\left[\mathrm{Ca}^{2+}\right]_{\mathrm{i}}$ during illumination was monotonic. After the illumination was terminated, $\left[\mathrm{Ca}^{2+}\right]_{i}$ rose again to its time zero value.

Our observation that the $\left[\mathrm{Ca}^{2+}\right]_{\mathrm{i}}$ of the rod falls during illumination emphasizes the importance of measuring $\left[\mathrm{Ca}^{2+}\right]_{\mathrm{i}}$ rather than inferring it from indirect measurements. The discovery of a light-induced rise in external $\mathrm{Ca}^{2+}$ (Gold and Korenbrot, 1980 , 1981; Yoshikami et al., 1980) was initially attributed to an extrusion from the rod of excess $\left[\mathrm{Ca}^{2+}\right]$ that had been released into the cytosol from the disks. Bownds (1980, 1981), however, pointed out that external $\left[\mathrm{Ca}^{2 \dagger}\right]$ would also rise if illumination directly stimulated a net efflux of $\mathrm{Ca}^{2+}$ from the rod, though in that case it would be associated with a fall in $\left[\mathrm{Ca}^{2+}\right]_{i}$, rather than with a rise. Our results demonstrate that this latter alternative is correct. Indeed, they are consistent with the view, outlined below, that $\left[\mathrm{Ca}^{2+}\right]_{\mathrm{i}}$ is determined entirely by the balance between $\mathrm{Ca}^{2+}$ influx through the light-sensitive channels and its extrusion by the $\mathrm{Na} / \mathrm{Ca}$ exchanger.

In prolonged darkness, the rod is in a steady state. Ionic concentrations on either side of the plasma membrane are constant, the influx of $\mathrm{Ca}^{2+}$ through the light-sensitive channels being exactly balanced by its efflux. Upon exposure to a bright light, the closure of light-sensitive channels greatly reduces the influx of $\mathrm{Ca}^{2+}$. The $\mathrm{Na} / \mathrm{Ca}$ exchanger, whose rate is determined by $\left[\mathrm{Ca}^{2+}\right]_{i}$, continues to expel $\mathrm{Ca}^{2+}$, however, causing a fall in free $\left[\mathrm{Ca}^{2+}\right]_{i}$, a consequent slowing of the exchange rate, and the eventual establishment of a new steady state. Suction-pipette measurements of the transient $\mathrm{Na} / \mathrm{Ca}$ exchange current in toad rods (Yau and Nakatani, 1985a) showed that it declines exponentially following exposure to a saturating light, with a time constant of only $0.4 \mathrm{sec}$. The longer time constant of the decline in free $\left[\mathrm{Ca}^{2+}\right]_{\mathrm{i}}$ measured in the present experiments is not unexpected, however, given that fura- 2 acts as a high-affinity $\mathrm{Ca}$ buffer and therefore slows any change in free $\left[\mathrm{Ca}^{2+}\right]_{\mathrm{i}}$ in the ROS.

In the presence of a saturating light, which closes all lightsensitive channels, the new steady state will be established when the rates of influx and efflux of $\mathrm{Ca}^{2+}$ are exactly balanced. If, as we have assumed, the $\mathrm{Ca}^{2+}$ efflux is due entirely to a $\mathrm{Na} / \mathrm{Ca}$ exchanger with 3:1 stoichiometry, then, as Blaustein and Hodgkin (1969) pointed out, the exchanger will come into equilibrium when free $\left[\mathrm{Ca}^{2+}\right]_{\mathrm{i}}$ obeys the relation,

$$
\frac{\left[\mathrm{Ca}^{2+}\right]_{\mathrm{i}}}{\left[\mathrm{Ca}^{2+}\right]_{0}}=\frac{\left[\mathrm{Na}^{+}\right]_{\mathrm{i}}{ }^{3}}{\left[\mathrm{Na}^{+}\right]_{0}{ }^{3}} \cdot c^{V E / R T},
$$

where $V$ is the transmembrane potential. Equation 3 thus provides an independent means of predicting the level to which free $\left[\mathrm{Ca}^{2+}\right]_{i}$ should fall during exposure to a saturating light (see Hodgkin and Nunn, 1987).

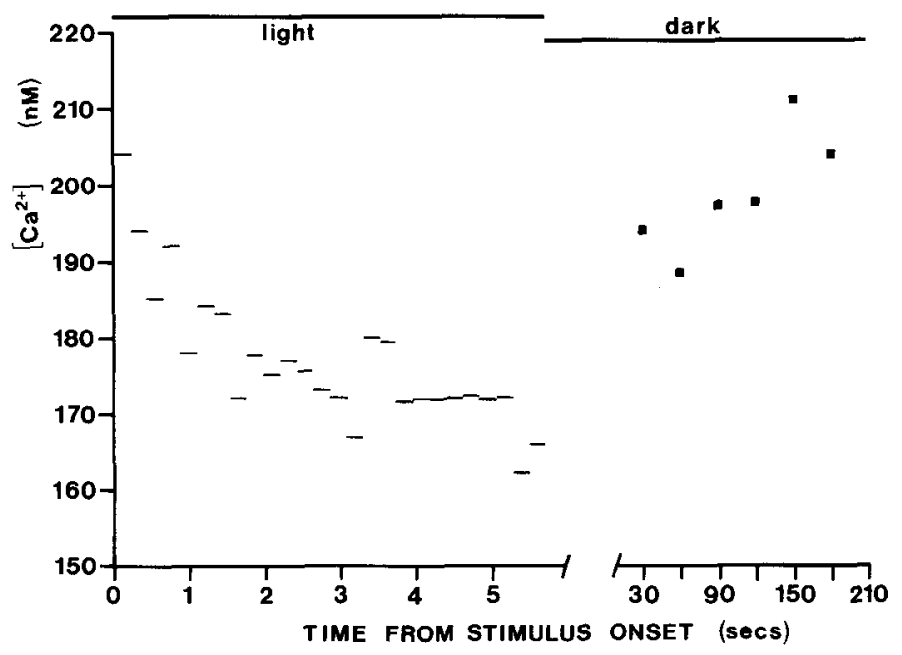

Figure 5. Recovery of resting free $\left[\mathrm{Ca}^{2+}\right]_{i}$ following extinction of the stimulus. The free $\left[\mathrm{Ca}^{2+}\right]_{\mathrm{i}}$ falls during the light response and rises again to its initial level when the light is turned off. The stimulus used in these measurements bleached 1840 rhodopsin molecules ${ }^{-1} \operatorname{rod}^{-1} \mathrm{sec}^{-1}$. 
In our experiments, $\left[\mathrm{Na}^{+}\right]_{0}=133 \mathrm{~mm}$ and $\left[\mathrm{Ca}^{3+}\right]_{0}=1.5$ mM. $\left[\mathrm{Na}^{+}\right]_{\mathrm{i}}$ is taken to be $\sim 12 \mathrm{~mm}$ in darkness (Torre, 1982). A saturating step of light causes the rod to hyperpolarize from a dark potential of $-40 \mathrm{mV}$ to a steady potential of $\sim-55 \mathrm{mV}$ (unpublished observations). Since $\mathrm{Na}^{+}$carries the major part of the dark current, this must be accompanied by a decrease in $\left[\mathrm{Na}^{+}\right]_{i}$, unless other major pathways for $\mathrm{Na}^{+}$entry are activated by the hyperpolarization. However, the rate at which $\left[\mathrm{Na}^{\prime}\right]_{i}$ turns over in darkness (Torre, 1982) is such that a fall of more than $1 \mathrm{~mm}$ during our $5.7 \mathrm{sec}$ stimulus is unlikely, and the actual fall may well be less than that. If we take $\left[\mathrm{Na}^{+}\right]_{\mathrm{i}}$ to be between 11 and $12 \mathrm{~mm}$ at the end of our saturating stimulus, then, from equation (3), we conclude that the exchanger should come into equilibrium when $\left[\mathrm{Ca}^{2+}\right]_{\mathrm{i}}$ is in the range $100-150 \mathrm{nM}$, in excellent agreement with our measured value. This agreement supports the notion that $\mathrm{Ca}$ is expelled from the ROS exclusively by the $\mathrm{Na} / \mathrm{Ca}$ exchanger.

While it is now known that the light-sensitive channels of the rod are gated by cGMP and not by $\mathrm{Ca}^{2+}$ (Fesenko et al., 1985, 1986; Nakatani and Yau, 1985; Yau and Nakatani, 1985b; Matthews, 1986), there is strong evidence that suggests that $\left[\mathrm{Ca}^{2+}\right]_{\mathrm{i}}$ may be important in regulating the state of adaptation of the rod (Torre et al., 1986; Hodgkin et al., 1987). The mechanism by which it does this is not understood, though it seems most likely that it involves a modulation of the rod's cGMP metabolism (Stryer, 1986). Both the synthesis of cGMP by the guanylate cyclase and its hydrolysis by the phosphodiesterase have been proposed as possible areas of action of $\mathrm{Ca}^{2+}$. Calcium is believed to inhibit the guanylate cyclase (Fleischman and Denisevich, 1979; Lolley and Racz, 1982; Hodgkin et al., 1985), and it has also been suggested that, directly or indirectly, it may activate the phosphodiesterase (Bownds, 1980; Robinson et al., 1980; Lamb, 1986; Torre et al., 1986). In either case, the observed light-induced fall in $\left[\mathrm{Ca}^{2+}\right]_{\mathrm{i}}$ would tend to restore the cGMP concentration to its dark level, thereby promoting light adaptation.

\section{References}

Beavo, J. A., N. L. Rogers, O. B. Crofford, J. G. Hardman, E. W. Sutherland, and E. V. Newman (1970) Effects of xanthine derivatives on lipolysis and on adenosine $3^{\prime}, 5^{\prime}$-monophosphate phosphodiesterase activity. Mol. Pharmacol. 6: 597-603.

Blaustein, M. P., and A. L. Hodgkin (1969) The effect of cyanide on the efflux of calcium from squid axons. J. Physiol. (Lond.) 200: 497527.

Bownds, M. D. (1980) Biochemical steps in visual transduction: Roles for nucleotides and calcium ions. Photochem. Photobiol. 32: 487490.

Bownds, M. D. (1981) Biochemical pathways regulating transduction in frog photoreceptor membranes. Curr. Top. Membr. Trans. 15: 203214.

Capovilla, M., L. Cervetto, and V. Torre (1982) Antagonism between steady light and phosphodiesterase inhibitors on the kinetics of rod photoresponses. Proc. Natl. Acad. Sci. USA 79: 6698-6702.

Capovilla, M., L. Cervetto, and V. Torre (1983) The effect of phosphodiesterase inhibitors on the electrical activity of toad rods. $J$. Physiol. (Lond.) 343: 277-294.

Cervetto, L., and P. A. McNaughton (1986) The effects of phosphodiesterase inhibitors and lanthanum ions on the light-sensitive current of toad retinal rods. J. Physiol. (Lond.) 370: 91-100.

Connor, J. A. (1986) Digital imaging of free calcium changes and of spatial gradients in growing processes in single, mammalian central nervous system cells. Proc. Natl. Acad. Sci. USA 83: 6179-6183.

Fcin, A., and E. Szuts (1982) Photoreceptors: Their Role in Vision, Cambridge U. P., Cambridge, UK.
Fesenko, E. E., S. S. Kolesnikov, and A. L. Lyubarsky (1985) Induction by cGMP of cationic conductance in the plasma membrane of retinal rod outer segment. Nature 313: 310-313.

Fesenko, E. E., S. S. Kolcsnikov, and A. L. Lyubarsky (1986) Direct action of cGMP on the conductance of retinal rod plasma membrane. Biochim. Biophys. Acta 856: 661-671.

Fleischman, D., and M. Denisevich (1979) Guanylate cyclase of isolated bovine retinal rod axonemes. Biochemistry 18: 5060-5066.

Gold, G. H., and J. I. Korenbrot (1980) Light-induced calcium release by intact retinal rods. Proc. Natl. Acad. Sci. USA 77: 5557-5561.

Gold, G. H., and J. I. Korenbrot (1981) The regulation of calcium in the intact retinal rod: A study of light-induced calcium release by the outer segment. Curr. Top. Membr. Transp. 15: 307-330.

Grynkiewicz, G., M. Poenie, and R. Y. Tsien (1985) A new generation of $\mathrm{Ca}^{2+}$ indicators with greatly improved fluorescence properties. $\mathrm{J}$. Biol. Chem. 260: 3440-3450.

Hamm, H. E., and M. D. Bownds (1986) Protein complement of rod outcr scgments of frog retina. Biochemistry 25: 4512-4523.

Hodgkin, A. L., and B. J. Nunn (1987) The effect of ions on $\mathrm{Na}-\mathrm{Ca}$ exchange in salamander rods. J. Physiol. (Lond.) 391: 370-398.

Hodgkin, A. L., P. A. McNaughton, and B. J. Nunn (1985) The ionic selectivity and calcium dependence of the light-sensitive pathway in toad rods. J. Physiol. (Lond.) 358: 447-468.

Hodgkin, A. L., P. A. McNaughton, and B. J. Nunn (1987) Measurement of sodium-calcium exchange in salamander rods. J. Physiol. (Lond.) 391: 347-370.

Lakowicz, J. R. (1983) Principles of Fluorescence Spectroscopy, Chapt. 10, Plenum, New York.

Lamb, T. D. (1986) Transduction in vertebrate photoreceptors: The roles of cyclic GMP and calcium. Trends Neurosci. 9: 224-228.

Lolley, R. N., and E. Racz (1982) Calcium modulation of cyclic GMP synthesis in rat visual cells. Vision Res. 22: 1481-1486.

Matthews, G. (1986) Comparison of the light-sensitive and cyclic GMP-sensitive conductances of the rod photoreceptor: Noise characteristics. J. Neurosci. 6: 2521-2526.

McNaughton, P. A., L. Cervetto, and B. J. Nunn (1986) Measurement of intracellular free calcium concentration in salamander rods. Nature 322: 261-263.

Mullins, L. J., and J. Requena (1979) Calcium measurement in the periphery of an axon. J. Gen. Physiol. 74: 393-413.

Nakatani, K., and K.-W. Yau (1985) cGMP opens the light-sensitive conductance in retinal rods. Biophys. J. 47: 356a.

Robinson, P. R., S. Kawamura, B. Abramson, and M. D. Bownds (1980) Control of the cyclic GMP phosphodiesterase of frog photoreceptor membranes. J. Gen. Physiol. 76: 631-645.

Schröder, W. H., and G. L. Fain (1984) Light-dependent calcium release from photoreceptors measured by laser micro-mass analysis. Nature 309: 268-270.

Sickel, W. (1965) Respiratory and electrical responses to light stimulation in the retina of the frog. Science 148: 648-651.

Somlyo, A. P., and B. Walz (1985) Elemental distribution in Rana pipiens retinal rods: Quantitative electron probe analysis. J. Physiol. (Lond.) 358: 183-195.

Steinemann, A., and L. Stryer (1973) Biochemistry 12: 1499-1502.

Stryer, L. (1986) Cyclic GMP cascade of vision. Annu. Rev. Neurosci. 9: $87-119$.

S7uts, E. Z., and R. A. Cone (1977) Calcium content of frog rod outer segments and disks. Biochim. Biophys. Acta 468: 194-208.

Torre, V. (1982) The contribution of the electrogenic sodium-potassium pump to the electrical activity of toad rods. J. Physiol. (Lond.) 333: 315-341.

Torre, V., H. R. Matthews, and T. D. Lamb (1986) The role of calcium in regulating the cyclic GMP cascade of phototransduction. Proc. Natl. Acad. Sci. USA 83: 7109-7113.

Yau, K.-W., and K. Nakatani (1984) Electrogenic Na-Ca exchange in retinal rod outer segment. Nature 311: 661-663.

Yau, K.-W., and K. Nakatani (1985a) Light-induced reduction of cytoplasmic free calcium in retinal rod outer segment. Nature 313: 579-582.

Yau, K.-W., and K. Nakatani (1985b) Light-suppressible, cGMP-sensitive conductance in the plasma membrane of a truncated rod outer segment. Nature 317: 252-255.

Yoshikami, S., J. S. George, and W. A. Hagins (1980) Light-induced calcium fluxes from the outer segment layer of vertebrate retinas. Nature 286: 395-398. 